\title{
Im Blickwinkel der Technik: Neue Verhältnisse von Wissenschaftstheorie und Wissenschaftsgeschichte
}

\author{
Alfred Nordmann
}

Berichte zur Wissenschaftsgeschichte, Bd. 35:3 (2012), S. 200-216

Summary: Changing Perspectives - From the Experimental to the Technological Turn in History and Philosophy of Science. In the 1960s the philosophy of science was transformed through the encounter with the history of science, resulting in a collaborative venture by the name of "History and Philosophy of Science" (HPS). Philosophy of science adopted ever more regularly the format of the case study to reconstruct certain episodes from the history of science, and historians were mostly interested in the production of scientific knowledge. The so-called "experimental turn" of the 1980s owed to this interaction between philosophy and history. Its guiding question remained quite traditional, however, namely "How do the sciences achieve an agreement between representation and reality?" Only the answers to this question broke with tradition by focusing not on theory but on the role of instruments and experiments. - Roughly 30 years after the experimental turn, another transformative encounter appears to be taking place. HPS is being transformed in the encounter with philosophy of technology. From the point of view of philosophy of technology, the question does not arise whether and how the agreement of mind and world, representation and reality can be achieved. When things are constructed, built or made, human thinking and physical materiality are inseparably intertwined. Instead of seeking to describe a mind-independent reality, technoscientific researchers are working to acquire and demonstrate capabilities of experimental or predictive control. When science is regarded as a kind of technology, a program of study opens up for epistemology and so do avenues for the historiography of science. History of science might now show how the problems and procedures of the sciences arise from and impinge back upon a world that is itself a product of science and technology. It thereby abandons its traditional HPS niche existence and joins forces with environmental history, history of technology, social, labor, and consumer history.

Keywords: science and technoscience, history of the philosophy of science, philosophy of experiment, philosophy of technology, historiography of science, Ian Hacking,

Schlüsselwörter: Wissenschaft und Technowissenschaft, Geschichte der Wissenschaftstheorie, Philosophie des Experiments, Technikphilosophie, Wissenschaftsgeschichte, Ian Hacking 
Jede akademische Disziplin hat ihre Ursprungsmythen, ihre Leitfragen, ihr Traditionsbewusstsein, ihre heuristisch unverzichtbaren Trägheitsmomente. Natürlich gilt dies auch für die Wissenschaftsphilosophie. ${ }^{1}$ Und obgleich es kaum eine wissenschaftsphilosophische Position gibt, die unangefochten geblieben ist, obgleich sich die Wissenschaftsphilosophie gegenüber den Ansprüchen der Wissenschaftsgeschichte und der Soziologie wissenschaftlichen Wissens behaupten und ihren Frieden schließen musste, obgleich sie eine Wendung zu den Einzelwissenschaften, zum Experimentalismus, zur Laborpraxis und zur Praxis der Theoriebildung vollzogen hat, verfolgt sie hartnäckig auch weiterhin eine Hauptfrage, die ihren Blick auf heutige Forschung und die Geschichte neuzeitlicher Wissenschaft verengt. Ganz allgemein formuliert lautet diese erkenntnistheoretische Hauptfrage so: Wie weisen die Wissenschaften die Übereinstimmung von Darstellung und Wirklichkeit nach? So facettenreich und kontrovers diese Frage diskutiert werden kann, hat sie auch weite Teile der Wissenschaftsgeschichte in Haft genommen. Angesichts der resultierenden Engführung des Blicks könnte sich somit die eigentlich willkommene intime Beziehung von Wissenschaftsphilosophie und Wissenschaftsgeschichte als eine tödliche Umarmung für die Wissenschaftsgeschichte erweisen. ${ }^{2}$

Um diese Engführung zu verdeutlichen, wird im Folgenden von einem Konkurrenzprojekt die Rede sein - ein Projekt, das die bisherige Wissenschaftsphilosophie gewissermaßen vom Kopf auf die Hände stellt, indem es Wissenschaft als Technik versteht. Es präsentiert sich einerseits als ein komplementäres Projekt und versucht die klassische Wissenschaftsphilosophie keineswegs abzulösen. Andererseits artikuliert es einen Forschungsbegriff, der eine aus dem 19. und 20. Jahrhundert überlieferte normative Wissenschaftsauffassung zutiefst erschüttert und unbedingt von ihr abgewehrt werden muss. Nicht weniger als das Verhältnis von Wissenschaft und Aufklärung steht dabei auf dem Spiel und die Idee, dass in der Wahrheitssuche ein kritisches Moment steckt, das dem menschlichen Gestaltungswillen

\footnotetext{
${ }^{1}$ Keine akademische Disziplin kann es sich leisten, ihre eigenen Voraussetzungen kritisch zu reflektieren - dies wäre schon eine Krisenerscheinung, die normalwissenschaftliches Forschen in Frage stellt. Dass auch dies für die Wissenschaftsphilosophie gilt, überrascht schon eher. Selbst dort, wo sich Wissenschaftsphilosophie selbst-reflexiv historisch wendet und zur Geschichte der Wissenschaftsphilosophie wird - ihrerseits vielleicht eine Krisenerscheinung -, bestätigt und verhärtet sie nur ihre Ausgangsvoraussetzungen. Dies verdankt sich insbesondere dem Einfluss Michael Friedmans, siehe Alfred Nordmann, Another Parting of the Ways: Intersubjectivity and the Objectivity of Science, Studies in History and Philosophy of Science 43, 1 (2012), 38-46 und Michael Friedman, Reconsidering the Dynamics of Reason: Response to Ferrari, Mormann, Nordmann, and Uebel, Studies in History and Philosophy of Science 43, 1 (2012), 47-53.
}

${ }^{2}$ Letztere These wird erst am Schluss dieses Textes weiter ausgeführt. Sie verdankt sich Diskussionen mit Ann Johnson. 
Grenzen zu setzen vermag. Dieses Konkurrenzprojekt könnte Technowissenschaftsphilosophie heißen und sein Ausgangspunkt ist, dass alle Wissenschaft Technik und somit Technowissenschaft ist und dass Technowissenschaft aus der Perspektive der Technikphilosophie betrachtet werden muss. Ganz allgemein formuliert würde ihre Hauptfrage ganz anders lauten als die nach Übereinstimmung von Darstellung und Wirklichkeit: Sofern es den Technowissenschaften gar nicht um die Darstellung der Wirklichkeit geht, was ist das Verhältnis von Wissen und Können in den vielfältigen Fertigkeiten des Bauens und Machens, des Manipulierens und Modellierens? Tradierte Begriffe wie Erklären und Verstehen, Validierung und Objektivität müssten nun so verstanden werden, wie sie für technische Konstruktionsverfahren oder technowissenschaftliche Phänomenbeherrschung gelten. Und obgleich technische Konstruktionen natürlich auch gesellschaftlichen Fortschritt befördern können, stellen wir uns in der Regel nicht vor, dass sie eine aufklärerische Idee enthalten, einen kritischen Impuls geben oder mit dem Schwert der Wahrheit einer angemaßten Macht mutig entgegentreten.

Auf das Verhältnis von Wissenschaftsphilosophie und Wissenschaftsgeschichte bezogen lautet die hier vertretene These durchaus ambivalent so: Die de facto stattfindende Preisgabe eines aus normativen und kulturellen Gründen eigentlich unverzichtbaren Wissenschaftsideals ermöglicht ein freieres und produktiveres Verhältnis von Philosophie und Geschichte technowissenschaftlich aufgefasster Forschung. Dieser Ambivalenz stellen kann sich aber nur, wer zunächst einmal bereit ist, sich auf das Programm einer Technowissenschaftsphilosophie einzulassen. ${ }^{3}$ Hierzu verfahre ich in fünf Schritten. Ausgangspunkt ist der von Ian Hacking eingeleitete „experimental turn“, der zwar das Verhältnis von Eingriff und Darstellung beleuchtet, das klassische Wissenschaftsideal aber keineswegs in Frage stellt. Nachdem die Differenz von Wissenschaft und Technowissenschaft kurz skizziert wurde, zeige ich im dritten Abschnitt, dass verschiedene Ansätze der heutigen Wissenschaftstheorie dieses Ideal zwar nicht in Frage stellen, aber doch aushöhlen, indem sie sich zunehmend eines technischen Idioms bedienen, um die Übereinstimmungen von Darstellung und Wirklichkeit zu rekonstruieren. Dann erst werden im vierten Abschnitt die neuen Verhältnisse sichtbar, die sich aus einer explizit technikphilosophischen Perspektivierung wissenschaftlicher

\footnotetext{
${ }^{3}$ Diese Ambivalenz spiegelt sich im Bewusstsein vieler Wissenschaftler, aber beispielsweise auch im Schriftenverzeichnis des Autors: Ungefähr die Hälfte widmet sich historisch und systematisch dem klassischen Bild von Wissenschaft als theoretischer Weltbeschreibung, die andere Hälfte der technowissenschaftlichen Forschungspraxis und ihrer Wissensproduktion.
} 
Forschungspraxis ergeben. Diese werden abschließend auf ihre historiographischen Konsequenzen hin diskutiert.

\section{IAN HACKING UND DIE AUSGEBLIEBENEN FOLGEN}

Kaum ein Buch war so einflussreich für die Wissenschaftsphilosophie der letzten 30 Jahre wie Ian Hackings Representing and Intervening oder Einführung in die Philosophie der Naturwissenschaften. Es hat die Leitfrage der Wissenschaftsphilosophie problematisiert, es nimmt technisches Handeln wissenschaftsphilosophisch ernst und begründete mit der ,philosophy of scientific experiment' einen neuen Forschungszweig. Trotz alledem und seinem Plädoyer für eine grundlegende Reform der Wissenschaftsphilosophie führt es aber das klassische Projekt der Wissenschaftsphilosophie einschließlich seiner Leitfrage fort, und so sehr es den technischen Eingriff des Experimentators betont, sieht auch dieses Buch in der Technik nicht mehr als ein untergeordnetes Hilfsmittel und bloßes Instrument zur Realisierung eines geistigen Ziels.

„Indem wir uns ausschließlich mit der Erkenntnis als Darstellung der Natur beschäftigen“, schreibt Hacking, „fragen wir uns, wie es uns je gelingen kann, den Darstellungen zu entrinnen und uns an der Welt festzuhaken“.4 Mit dieser Formulierung spricht Hacking die Hauptfrage der überlieferten Wissenschaftsphilosophie an und kritisiert sie offenbar auch schon. Wie wir sehen werden, kritisiert er aber nur eine spezielle Auslegung dieser Frage und lässt sie in ihrer allgemeinen Fassung stehen.

Zwischen die beiden der Darstellung und dem Eingriff gewidmeten Hauptteile seines Buches hat Hacking ein „Intermezzo“ eingefügt, mit dem er den Leser davor warnt, einen Gegensatz von Darstellung und Eingriff anzunehmen. Nicht gegen das wissenschaftsphilosophische Interesse an der Darstellung wende er sich, sondern gegen die Auffassung, dass Darstellung etwas bloß Gedankliches oder Theoretisches sei. Und so kritisiert er die so genannte Zuschauertheorie der Erkenntnis vor allem, weil sie nicht einbezieht, dass Darstellungen etwas Gemachtes, auch mittels Eingriff Erzeugtes sind. Diese Zuschauertheorie drängt sich auf, wenn das Verhältnis von Darstellung zum Dargestellten auf das Verhältnis von Theorie und Welt reduziert wird:

\footnotetext{
${ }^{4}$ Ian Hacking, Einführung in die Philosophie der Naturwissenschaften, Stuttgart: Reclam 1996, S. 219.
} 
Die Begriffe Inkommensurabilität, transzendentaler Nominalismus, Wahrheitsersatz und Denkstil gehören zur philosophischen Fachsprache. Sie stellen sich ein, wenn man sich Gedanken macht über das Verhältnis von Theorie und Welt. Sie alle führen in eine idealistische Sackgasse. Keiner gibt den Anstoß zur Ausbildung eines gesunden Realitätssinns. John Dewey, ein Philosoph unseres eigenen Jahrhunderts, hat boshaft von einer Zuschauertheorie der Erkenntnis gesprochen, von der die abendländische Philosophie besessen gewesen sei. Sofern wir bloße Zuschauer im Theater des Lebens sind, fragt es sich, wie wir anhand innerer Kriterien der vorüberziehenden Vorführung jemals in Erfahrung bringen können, was eine bloße Darstellung von Seiten der Schauspieler und was Wirklichkeit ist. 5

Dewey und Hacking sind nicht die einzigen, die entlarvend diagnostizieren oder spöttisch belächeln, dass die abendländische Philosophie besessen fragt, wie sich ein erkennendes Subjekt mit der Welt verhaken kann, wenn es erst einmal seinen Platz im Zuschauerraum eingenommen hat. Martin Heidegger und Hannah Arendt haben diese Besessenheit als eine grundlegende Verirrung der Neuzeit kulturkritisch an den Pranger gestellt: Erst zieht sich das Individuum in das Gehäuse seines Wahrnehmungsapparats und letztlich seines Schädels zurück, um sich dann zu fragen, wie es eine Übereinstimmung zwischen Innen und Außen, zwischen Geist und Welt, zwischen Theorie und Wirklichkeit geben kann. Diese Fragen nach Möglichkeit, Erzeugung und Überprüfung einer Übereinstimmung würden ohne diesen Rückzug gar nicht entstehen, was mit besonderer Deutlichkeit René Descartes vorgeführt hat. Seine Philosophie und seine Wissenschaft fangen ausdrücklich mit der in gewisser Hinsicht absurden Anstrengung an, sich am Kamin einzurichten und erst einmal jedes Tagesgeschäft und alles Empirische wegzudenken, bis er sich selbst auf ein denkendes Ding, den reinen Zuschauer reduziert hat. ${ }^{6}$ Sein philosophisches Gedankenexperiment - und mehr sollte es doch eigentlich nicht sein - rief das Bild eines vom Chaos der Sinneseindrücke überwältigten Menschen auf, der einen archimedischen Punkt finden muss, von dem aus Ordnung in das Chaos gebracht und die Welt verständlich werden kann. Dementsprechend erscheint wissenschaftliche Forschung als Produktion und Interpretation von Daten: Beobachtung und Experiment konfrontieren Wissenschaftler mit Eindrücken und Messungen, die mit Hilfe einer Theorie oder eines Modells zusammengefasst werden und möglichst Voraussagen beinhalten, die eine weitere Prüfung der Übereinstimmung von Theorie und Wirklichkeit ermöglichen.

Obwohl niemand bezweifeln wird, dass sich Descartes' Gedankenexperiment als außerordentlich fruchtbar für Wissenschaft und Philosophie erwiesen hat, ist auch nicht

\footnotetext{
${ }^{5}$ Hacking, Einführung (wie Anm. 4).

6 René Descartes, Discours de la méthode, Hamburg: Meiner 1997 und derselbe, Meditationen über die Grundlagen der Philosophie, Hamburg: Meiner 1993.
} 
schwer zu verstehen, wie artifiziell diese Konfrontation von Wirklichem und Gedanklichem, von zu erkennender Welt und erkennendem Subjekt, Datenquelle und Interpretationsraster ist. Insbesondere für die Technikphilosophie kann diese Konfrontation aus zweierlei Grund nicht als Ausgangspunkt dienen.

Zunächst mochte es vielleicht eine Zeit gegeben haben, zu der Naturerscheinungen und Sinneseindrücke eine verwirrende Mannigfaltigkeit aufwiesen und allein mit geistigen Mitteln geordnet werden konnten. Inzwischen sind aber mehrere tausend Jahre Wissenschafts- und Technikgeschichte vergangen, so dass heutige Menschen in einer weitgehend entzauberten, das heißt intellektualisierten, berechenbaren, technisierten, handlungsstrukturierenden Welt leben. Heute bedarf es eines hohen technischen Aufwands, um eine unverstandene und interpretationsbedürftige Vielfalt von Erscheinungen überhaupt noch zu produzieren, etwa in wissenschaftlichen Experimenten, in denen unerhörte Datenmengen generiert werden. Aber insofern diese Erscheinungen mit den Mitteln einer wissenschaftlich basierten Technik erzeugt und von technischen Medien aufgefasst und interpretiert werden, ist es mehr als seltsam, als Erkenntnisziel hier noch die Überbrückung zweier logisch unabhängig vorgestellter Sphären von Geist und Welt, Theorie und Wirklichkeit zu postulieren. Stattdessen treffen hier zwei aufeinander abgestimmte Apparate aufeinander, nämlich ein wissenschaftlich-technisches Experimentalsystem einschließlich der darin erzeugten Datensätze und ein wissenschaftlichtechnisches Wahrnehmungs-, Begriffs- und Denkinstrumentarium. Genauso rekonstruieren etwa Heidegger und Arendt die Anfänge der neuzeitlichen Wissenschaft bei Galileo und Newton, und genauso rekonstruieren sie die Erkenntnistheorie von Descartes über Kant bis Heisenberg. Beide zitieren Heisenbergs Aussage, der Mensch würde sich überall nur noch selbst begegnen. Während Arendt dabei an Galileos Verlegung des archimedischen Punktes in das erkennende Subjekt mit seinen Mess- und Beobachtungsapparaturen denkt, führt Heidegger dies auf die Konstruktion in Newtons Mechanik einer durchgängigen Natur zurück, die der mathematischen Darstellung von Ereignisfolgen dient. ${ }^{7}$

Es gibt einen zweiten Grund, warum es aus der Perspektive von Technik und Technikphilosophie nicht plausibel ist, die theoriebildende, dateninterpretierende, deutende Forschung einer geistesunabhängig konzipierten Wirklichkeit gegenüber zu stellen. Es ist

\footnotetext{
${ }^{7}$ Hannah Arendt, Vita Activa, München: Piper 1981, S. 255 f. und S. 364; Martin Heidegger, Die Frage nach der Technik, in: derselbe, Gesamtausgabe Bd. 7. Vorträge und Aufsätze, Frankfurt: Klostermann 2000, S. 28 und derselbe, Die Frage nach dem Ding: Zu Kants Lehre von den transzendentalen Grundsätzen, in: derselbe, Gesamtausgabe Bd. 41, Frankfurt: Klostermann 1984, S. 93 und 89.
} 
nämlich nicht so, dass wissenschaftliche Forschung ausschließlich oder vornehmlich darin besteht, Theorien, Hypothesen, Modelldarstellungen $\mathrm{zu}$ produzieren, also sprachliche Aussagen, die dann auf Wahrheit und Falschheit oder empirische Adäquatheit geprüft werden. Wer etwas zum Funktionieren bringt, der stellt keine Wahrheitsbehauptung auf, sondern erstellt ein technisches System, das an seiner Leistung gemessen wird, also an dem, was es kann und was es gewährt. Freilich gibt es technische Systeme, die eine korrekte Repräsentation der Wirklichkeit voraussetzen - der Wissenschaftsphilosoph Michael Friedman würde hier beispielsweise den Kalender nennen, der auf genauer astronomischer Kenntnis basiert. ${ }^{8}$ Schon ein Uhrwerk jedoch repräsentiert nichts oder allenfalls sich selbst, auch wenn sich ihm eine repräsentierende Funktion zuweisen lässt, wenn es etwa als Metapher oder Modell für Naturphänomene dienen soll. Und so werden in der Forschung bisweilen theoretische Aussagen formuliert, bisweilen Dinge zum Funktionieren gebracht, bisweilen Ereignisse experimentell erzeugt und stabilisiert, bisweilen interessante Objekteigenschaften und erworbene Fertigkeiten nachgewiesen, „proofs of concept“ etabliert. Wer heute in den wissenschaftlichen Zeitschriften blättert, findet natürlich immer noch Beiträge, in denen mittels neu erworbener Evidenz Hypothesen geprüft werden, findet vor allem aber Beiträge, in denen das kontrollierte Wachstum von Kohlenstoff-Nanoröhrchen oder die pharmazeutische Wirksamkeit eines Stoffs vorgeführt werden, in denen eine verbesserte Methode des physischen Eingriffs oder der Visualisierung demonstriert werden, in denen ein Modellsystem zum Laufen gebracht wird. Nun kann auch der Zuwachs an technischen Fertigkeiten als objektive Erkenntnis beschrieben werden und präsentiert sich in den genannten Zeitschriften auch so. Nach welchen Kriterien wir hier aber von Erkenntnis sprechen können, ist eine für die Philosophie notorisch schwierige Frage. Jedenfalls kommen wir nicht weit, wenn wir diese Erkenntnis als Übereinstimmung von Geist und Welt deuten, schon weil sich an einem Uhrwerk oder einer Computersimulation oder einer genetisch modifizierten Labormaus oder einem vorgeführten Wirkmechanismus Geist und Welt gar nicht unterscheiden lassen. ${ }^{9}$

\footnotetext{
${ }^{8}$ Michael Friedman, A post-Kuhnian Approach to the History and Philosophy of Science, The Monist 93 (2010), 495-515.

${ }^{9}$ Alfred Nordmann, Collapse of Distance: Epistemic Strategies of Science and Technoscience, Danish Yearbook of Philosophy 41 (2006), 7-34 und derselbe, Was wissen die Technowissenschaften?, in: Friedrich Gethmann (Hrsg.), Lebenswelt und Wissenschaft: Kolloquiumsband des XXI. Deutschen Kongresses für Philosophie, Hamburg: Meiner 2011, S. 566-579. - Seit Platon arbeitet die Philosophie mit der Definition von Erkenntnis als wahrer und gerechtfertigter Meinung (justified true belief). Wer den Nachweis erbringt, eine Fertigkeit erworben zu haben, formuliert jedoch keine Meinung, also einen Satz, der sich nun als wahr oder falsch, gerechtfertigt oder ungerechtfertigt erweisen könnte. Und noch einmal anders formuliert: Wer die Übereinstimmung von Geist und Welt sucht, muss sich permanent sorgen, ob es überhaupt möglich ist, mit geistigen Mitteln
} 
Aus technikphilosophischer Perspektive habe ich jetzt Deweys und Hackings Verwunderung angesichts einer Zuschauertheorie der Erkenntnis und ihrer artifiziellen Problemstellung zu verdeutlichen gesucht. An dieser Stelle, ließe sich vermuten, findet Hackings Übergang von „representing“ zu „intervening“ statt, wendet sich Hacking von Erkenntnis als Darstellung der Natur ab und einem an Technik orientierten Erkenntnisbegriff zu. Hacking jedoch verfährt anders, indem er sich allein gegen die unterstellte Passivität des Zuschauers wendet, gegen die Trennung von Denken und Handeln und gegen das „theoretische“, nur aufs Denken bezogene Interesse der Wissenschaftsphilosophie. ${ }^{10}$

Dabei glaube ich allerdings nicht, dass die Idee der Erkenntnis als Darstellung der Welt allein schon die Wurzel des Übels ist. Der Schaden rührt vielmehr daher, dass man sich auf Kosten des Eingreifens, Handelns und Experimentierens wie besessen mit nichts anderem beschäftigt als Darstellen, Denken und Theorie. ${ }^{11}$

So kritisiert Hacking also keineswegs die Vorstellung, dass wissenschaftliche Erkenntnis in der Darstellung und Abbildung der Natur besteht, sondern nur die darüber hinausgehende Auffassung, dass Darstellung und Abbildung der Natur eine Sache des bloß zuschauenden Denkens und nicht auch des Handelns sei. Nicht ,homo faber' sei der Mensch, sondern „ein darstellendes Wesen“, heißt es schon ein paar Seiten später. Wenn dieser ,homo depictor‘ an Felswände kritzelt und Figuren schnitzt, dann schaffe er immer Ebenbilder und formuliere damit „den Gedanken, daß diese Holzschnitzerei hier im Hinblick auf das Dargestellte etwas Wirkliches erkennen lässt“.12 Mit seiner Aufmerksamkeit auf „intervening“ und den experimentellen Eingriff schafft Hacking den Zuschauer ab und bezieht technisches Handeln in die Wissenschaftsphilosophie ein, tut dies aber nur auf Grund der Annahme, dass jede Präsentation eines Symbols oder Phänomens, auch die technische Stabilisierung eines Vorgangs im Labor, eine Wahrheitsbehauptung der korrekten Darstellung enthält, nämlich ein „dies hier ist wirklich“.13 Die Schwierigkeit und das Hauptproblem von Wissenschaft und

\footnotetext{
zu einer geistesunabhängigen Wirklichkeit vorzudringen. Dies ist die Sorge, dass sich die vermeintliche Wirklichkeit letztlich als Artefakt des begrifflichen und technischen Zugriffs erweisen könnte (hier ist „Artefakt“ also negativ konnotiert). Wer sich hingegen für einen therapeutischen Wirkstoff interessiert, will ein Artefakt er- zeugen und an diesem (positiv konnotierten) Artefakt nicht ausmachen, ob es sich nun einer geistesunabhängigen Wirklichkeit oder einem menschlichen Eingriff verdankt.

10 Hacking, Einführung (wie Anm. 4), S. 220 und 267.

11 Hacking, Einführung (wie Anm. 4), S. 220.

12 Hacking, Einführung (wie Anm. 4), S. 223, 228 f., 231 f.

13 Aus technikphilosophischer Perspektive erscheint Hackings anthropologisches „Märchen vom Ursprung der Sprache“ (Hacking, Einführung [wie Anm. 4], S. 228) ebenso merkwürdig wie die Zuschauertheorie der Erkenntnis: Wandmalereien und Holzschnitzereien sind bedeutsam und tragen zur praktischen Organisation des Zusammenlebens bei, auch ohne vornehmlich
} 
Wissenschaftsphilosophie bleibt demnach die Übereinstimmung der Darstellung mit etwas Dargestelltem. Hackings „experimental turn“ sieht die praktische Lösung dieses Problems im instrumentellen Realismus und somit darin, dass sich der Wirklichkeitsgehalt wissenschaftlicher Darstellungen schon dadurch erweisen lässt, dass in diesen Darstellungen ein experimenteller Eingriff, die technische Stabilisierung von Phänomenen oder die tätige Erzeugung von Effekten steckt. Insofern dieser instrumentelle Realismus dazu dient, das Realismusproblem zu lösen, bleiben die Instrumente und bleibt die Technik letztlich ein Hilfsmittel für die Erfassung der Wirklichkeit durch die Wissenschaft. Forschung wird von Hacking also nicht umfassend in ihren technischen Zusammenhang gestellt oder unter der Bedingung von Technik betrachtet. Wenn Phänomene im Labor geschaffen oder erzeugt werden, heißt dies für Hacking vornehmlich, dass es im Labor gelungen ist, das Ebenbild einer gesetzlich verfassten Natur herzustellen - ein technisches reproduzierbares Phänomen stellt die Gesetzmäßigkeiten dar, denen es sich verdankt. ${ }^{14}$

Wenn es noch eines weiteren Belegs bedürfte, dass Hacking immer noch die Leitfrage nach der Übereinstimmung von Darstellung und Wirklichkeit behandelt und diese nur neu beantwortet, dann ist es schließlich die eben genannte Einbettung seines Vorschlags in die Debatte über Realismus und Konstruktivismus. Tatsächlich lautet der erste Satz von Hackings Buch "Rationality and realism are the two main topics of today's philosophers of science“. ${ }^{15}$ Zusammen mit den Fragen des Physikalismus und der Einheit der Wissenschaften, der

\footnotetext{
als Behauptungen über das zu dienen, was wirklich sei, und ohne Debatten auszulösen der Art „Nein, nicht das, sondern dies hier ist wirklich“. In der Tat kann eine Holzschnitzerei nicht aussagen, dass etwas nicht existiert oder falsch repräsentiert ist, somit auch nicht, dass etwas existiert und richtig repräsentiert ist. Im Gespräch über Holzschnitzereien mag unter besonderen Bedingungen befunden werden, dass die eine „besser“ oder „ähnlicher“ sei als die andere (vorausgesetzt, dass beide die gleiche „Behauptung“ aufstellen und Darstellungen des gleichen Gegenstands überhaupt sein wollen), aber auch dann würde es noch kein Kriterium dafür geben, wann eine Schnitzerei etwas „Richtiges“ oder „Falsches“ über die Wirklichkeit „aussagt“. Dass Hacking diese Praxis den sprachlichen Theoriebildungen angleichen will, belegt vor allem, dass er aus den Realismusdebatten der traditionellen Wissenschaftsphilosophie heraus denkt und schreibt. Von einer theorie- und gedankenverliebten Philosophie hat sich nicht sehr weit entfernt, wer meint, dass durch die Erzeugung einer Holzfigur ein Gedanke formuliert wird (siehe hierzu Ludwig Wittgenstein, dessen Bildtheorie der Sprache auf der Entdeckung beruht, dass ein zunächst einmal gar nichts repräsentierendes Spiel mit Modellautos unter ganz besonderen Umständen so umfunktioniert werden kann, dass die Spielzeuge für tatsächliche Unfallfahrzeuge einstehen und so eine Modellsituation entsteht, die jetzt erst als Abbild einer Wirklichkeit gedeutet werden kann, siehe Ludwig Wittgenstein, Philosophische Betrachtungen. Philosophische Bemerkungen, (Ludwig Wittgenstein: Wiener Ausgabe Bd. 2, Wien: Springer 1994, S. 279).

${ }^{14}$ Hacking, Einführung (wie Anm. 4), S. 367-370.

15 Dieser Satz eröffnet den in der deutschen Ausgabe nicht übersetzten „analytical table of contents“ in Ian Hacking, Representing and Intervening, Cambridge: Cambridge University Press 1983, S. x.
} 
Inkommensurabilität und des wissenschaftlichen Fortschritts, internen und externen Einflussgrößen und rationalen Rekonstruktionen von Theoriendynamik gehört der Streit um Realismus und Konstruktivismus ganz gewiss in den Problemhorizont des ,homo depictor'. Angesichts dieser 1983 und noch lange danach kontrovers diskutierten Themenfelder stellt sich jedoch die Frage, was inzwischen eigentlich aus ihnen geworden ist: Welche Philosophen oder Historiker interessieren sich heute noch für Theorienwahl und ihre rationale Rekonstruktion, wer ereifert sich über die Frage, ob Biologie auf Physik reduzierbar sei, wen treibt der Konstruktivismus auf die Barrikaden, wer möchte die wirkliche Wirklichkeit von theoretischen Entitäten nachweisen und was sind also die Hauptfragen der heutigen Wissenschaftsphilosophie? Dies führt auf die Situation der Wissenschaftsphilosophie fast 30 Jahre nach Hacking.

\section{WISSENSCHAFT UND TECHNOWISSENSCHAFT}

Die ausführliche Auseinandersetzung mit Ian Hackings Representing and Intervening verdeutlicht, dass keineswegs schon alles getan ist, wenn das Wechselspiel von technischer Phänomenerzeugung und theoretischer Darstellung in den Blick genommen wird. Tatsächlich hat die Wissenschaftsphilosophie in den letzten Jahrzehnten ein technisches Beschreibungsvokabular herausgebildet, mit dem detailreich und differenziert aufgezeigt werden kann, wie die Übereinstimmung von Darstellung und Dargestelltem hergestellt wird. Während diese Beschreibungen ganz im Sinne Hackings ein technisches Bild theoretischer Praxis zeichnen, werden sie der technowissenschaftlichen Wissensproduktion nur ansatzweise gerecht und sagen noch gar nichts aus über den Zuwachs objektiven Wissens durch den Nachweis des kontrollierbaren Wachstums von Kohlenstoff-Nanoröhrchen. Wo nicht nach der unterstützenden Rolle von Technik für die Übereinstimmung von Darstellung und Wirklichkeit gefragt wird, sondern nach dem Wissensfortschritt, der im technischen Zugriff auf komplexe Phänomene und Prozesse steckt, bedarf es einer technikphilosophisch informierten Wissenschaftsphilosophie. ${ }^{16}$

\footnotetext{
16 Was hat die Technikphilosophie der Wissenschaftsphilosophie außer einem anderen Ausgangspunkt zu bieten? Dies bedarf einer qualifizierteren Antwort, die ich in diesem Zusammenhang nicht geben kann, die aber schließlich darauf hinausläuft, hier auch eine Herausforderung an die Technikphiloso- phie zu formulieren. Aufmerksame Leser werden jedenfalls feststellen, dass
} 
Um all dies zu verdeutlichen, zunächst eine kurze und verkürzende Charakterisierung von Wissenschaft und Technowissenschaft, wie sie sich aus ihrer bisher nur impliziten Kontrastierung ergibt. Diese Kontrastierung bietet den Hintergrund, vor dem einige jüngere Entwicklungen der Wissenschaftsphilosophie eingeordnet wer- den können, die über die klassischen Themen der Rationalität und des Realismus hinaus zu einem technischen Idiom vorgedrungen sind, somit selbst schon technowissenschaftlich vorgehen, dabei aber die technowissenschaftliche Forschung nicht reflektieren, sondern immer noch eine Praxis der Theoriebildung beschreiben.

Die Wissenschaften verfolgen die theoretische Darstellung einer geistesunabhängigen Wirklichkeit, sie müssen also dafür Sorge tragen, dass Theorie und Wirklichkeit in einer gehaltvollen Beziehung zueinanderstehen. Die Physik leistet dies auch heute noch auf exemplarische Weise und gilt nicht umsonst als Leitwissenschaft. Als Beispiel mag die Suche nach dem Higgs-Boson dienen, für die mit dem Large Hadron Collider ein gigantischer technischer Zusammenhang geschaffen wurde. Ob das Higgs-Boson nachgewiesen werden kann oder nicht, ist dezidiert keine technowissenschaftliche Frage, da es hier ganz klassisch um die Prüfung einer Hypothese oder Theorie geht, also um die wahre oder empirisch adäquate Darstellung der Wirklichkeit. Gerade weil der technische Aufwand hoch ist, kommt es ganz darauf an, dass die Evidenz für das Higgs-Boson eben kein technisches Artefakt ist, sondern einem Naturgeschehen zugeschrieben werden kann. Die gehaltvolle Beziehung von Theorie und Wirklichkeit ist dann gegeben, wenn die Evidenz durch unsere Begriffe, Methoden und den technischen Eingriff nur zum Vorschein gebracht, nicht aber verursacht wird. ${ }^{17}$ Als Suche nach dem Higgs-Boson und somit nach Evidenz für Theorienkritik und wahre Welterkenntnis steht Wissenschaft in der Tradition der Aufklärung. Dagegen erbringen die Technowissenschaften die Aneignung und den Nachweis von Fertigkeiten der Phänomenbeherrschung. Dies beinhaltet grundlegende Fertigkeiten der Modellierung und der Voraussage, der Visualisierung und Manipulation. Wenn beispielsweise gezeigt wird, dass das Wachstum von KohlenstoffNanoröhrchen gesteuert werden kann oder dass die Replikation einer Krebszelle durch einen

\footnotetext{
in diesen Seiten allenfalls die Fruchtbarkeit von Heideggers Technikphilosophie auch der modernen Naturwissenschaft zur Sprache kommt.

17 Dieser Art ontologischer Sorge bezüglich der Evidenz unseres Wirklichkeitswissens steht die ontologische Gleichgültigkeit von Bastlern, Ingenieuren oder Technowissenschaftlern gegenüber. Zur „ontological indifference“ siehe Peter Galison, The Pyramid and the Ring, Vortrag bei der Tagung der Gesellschaft für Analytische Philosophie (GAP), Berlin 2006 und (ohne expliziten Bezug auf „ontologische Gleichgültigkeit“) Lorraine Daston, Peter Galison, Objectivity, New York: Zone Books 2007, Kapitel 7, hier S. 393 und 414.
} 
Wirkstoff unterbunden wird oder die Fehlerraten eines Detektortyps am Large Hadron Collider besser berechnet werden können, geht es nicht darum, das Naturgegebene vom technisch Gemachten zu unterscheiden. Vielmehr steckt im technischen Machen das nötige Wissen, um einen komplexen, gleichermaßen „natürlichen“ als auch „menschgemachten“ Zusammenhang systematisch $\mathrm{zu}$ beeinflussen. ${ }^{18}$ Technowissenschaftliche Forschung ist somit durch eine technische Weise des Erkennens und durch ihren Objektbezug definiert und nicht etwa dadurch, dass sie keine Grundlagen und nur Anwendungen schaffe, oder dadurch, dass sie nur aus Nutzungsinteressen und nicht aus Neugier gespeist sei. Im Rahmen technowissenschaftlicher Forschung werden grundlegende Fertigkeiten unter hoch technisierten Forschungsbedingungen erworben und entwickelt, die oft eine Weiterentwicklung der Forschungstechnik beinhalten und gelegentlich auch in andere technische Herstellungszusammenhänge, so genannte Anwendungen, einfließen. Hier dienen dann Materialforschung oder Nanotechnologie als Leitwissenschaften, wobei aber alle Wissenschaften - einschließlich der Geistes- und Sozialwissenschaften - als Technowissenschaften aufgefasst werden können, insofern sie Fertigkeiten entwickeln, die zu gesellschaftlichen Problemlösungen beitragen, und sofern wir sie also aus ihren technischen Rahmenbedingungen und Aufgabenstellungen heraus verstehen. ${ }^{19}$

An diese noch oberflächliche Kontrastierung von Wissenschaft und Technowissenschaft schließen sich nun zwei Beobachtungen zu gegenwärtigen Tendenzen der Wissenschaftsphilosophie an, denen die nächsten beiden Abschnitte gewidmet sind. Erstens bedient sich die Beschreibung der wissenschaftlichen Anstrengung um Übereinstimmung von Theorie und Wirklichkeit zunehmend eines technisch-ingenieurhaften Idioms. Und obwohl damit, zweitens, Wissenschaft noch nicht als Technik oder Technowissenschaft gesehen wird, gibt es auch hierfür erste Ansätze, ohne dass jetzt schon von einer bereits etablierten Technowissenschaftsphilosophie gesprochen werden könnte.

\footnotetext{
18 Schon Peter Galisons Image and Logic, Chicago: University of Chicago Press 1997, hat gezeigt, dass Forschungsstätten wie der Large Hadron Collider zwar ganz auf die Prüfung einer Theorie ausgerichtet und somit klassisch wissenschaftlich orientiert sein mögen, dass dort aber doch nur ein Bruchteil der Forschung in diesem Sinne wissenschaftlich ist und ein Großteil der Arbeit beispielsweise mit der Entwicklung und Modellierung von Detektoren befasst ist, also technowissenschaftlich mit der Aneignung und dem Nachweis von grundlegenden Beobachtungsfertigkeiten.

19 Die Unterscheidung von Wissenschaft und Technowissenschaft kann noch ausgeführt und philosophisch tiefer gelegt werden, siehe hierzu etwa Bernadette Bensaude-Vincent, Sacha Loeve, Alfred Nordmann, Astrid Schwarz, Matters of Interest: The Objects of Research in Science and Technoscience, Journal for General Philosophy of Science 42 (2011), 365-383. Hier werden auch methodologische Fragen zum Verhältnis dieser beiden Begriffe weiter verfolgt.
} 


\section{KLASSISCHE WISSENSCHAFTSPHILOSOPHIE IM NEUEN IDIOM DER TECHNIK}

Die erste Beobachtung lässt sich mit einer kurzen Liste von drei gegenwärtig diskutierten Ansätzen untermauern, die wissenschaftliche Praxis in einem technischen Idiom beschreiben. Da sind zunächst die von Margaret Morrison oder Nancy Cartwright vorgeschlagenen Rekonstruktionen wissenschaftlichen Modellierens. ${ }^{20}$ Im Vordergrund stehen hierbei Vorstellungen des "fittings" und "tunings": Modelle dienen dazu, eine Passung zu erzeugen zwischen Theorien, Prinzipien oder Begriffen einerseits, Phänomenen andererseits. Hier handelt es sich um lokale Feinabstimmungen, wobei sich das Modell als plastisches Werkzeug, Medium oder Mittel erweist, um besondere Bedingungen mit allgemeinen Prinzipien zu verkoppeln. Und während es sich bei „fitting" und „tuning" um technische Vorgehensweisen handelt, dienen sie ganz im Sinne der wissenschaftsphilosophischen Leitfrage der Konstruktion zumindest lokaler Übereinstimmungen von Darstellung und Wirklichkeit.21 Ähnlich einflussreich und stimulierend erweist sich derzeit der Vorschlag, dass eine wissenschaftliche Erklärung darin besteht, einen Mechanismus zu spezifizieren, der ein Phänomen oder einen Prozess erzeugt. ${ }^{22}$ Dies ist ein weitreichender Vorschlag, insofern hier ganz entgegen der philosophischen Tradition von „Erklärung“ die Rede ist ohne Bezug auf Theorien, Modelle, Verallgemeinerbarkeit oder die logischen Beziehungen zwischen Sätzen. Der klassischen Frage nach Übereinstimmung von Darstellung und Dargestelltem ist diese Auffassung nur noch insofern verpflichtet, als es darum geht, eine wirkliche Struktur, den wirklichen Mechanismus aufzuweisen und nachvollziehbar zu repräsentieren. Wir sollen sehen können, dass das wirkliche Geschehen dem identifizierten Mechanismus entspricht. Im dritten Ansatz für den

\footnotetext{
${ }^{20}$ Nancy Cartwright, The Dappled World: A Study of the Boundaries of Science, Cambridge: Cambridge University Press 1999; Margaret Morrison, Models as Autonomous Agents, in: Mary Morgan, Margaret Morrison (Hrsgg.), Models as Mediators, Cambridge: Cambridge University Press 1999, S. 38-65.

21 Zurückverfolgen lässt sich dieses Motiv auf Ludwig Wittgenstein, Appendic C: How Can ,Knowing' Fit a Physical Fact?, in: derselbe, Philosophical Occasions, 1912-1951, herausgegeben von James C. Klagge und Alfred Nordmann, Indianapolis: Hackett Publishing 1993, S. 422-426. - In Verbindung mit einem anderen Modellbegriff und einer aus dem Software Engineering entlehnten iterativen Methode kann das „fitting“ und „tuning“ auch dazu dienen, nicht etwa eine mit der Wirklichkeit übereinstimmende Darstellung zu erzeugen, sondern ein technisches Modellsystem, das die Wirklichkeit substituiert. Die Unterscheidung von repräsentierenden und substituierenden Modellen kann hier nicht durchgeführt werden, gehört aber zu den Aufgaben der Technowissenschaftsphilosophie, vgl. etwa Johannes Lenhard, Mit allem rechnen, Habilitationsschrift, Universität Bielefeld 2011.

22 Der klassische Text hierzu ist von Peter Machamer, Lindley Darden, Carl Craver, Thinking About Mechanisms, Philosophy of Science 67 (2000), 1-25. Die technische Bedeutung der hier diskutierten Mechanismen ergibt sich aus Stuart Glennan, Mechanisms, Models, and Causation, Ph.D. Dissertation, University of Chicago 1992.
} 
Einsatz eines technischen Idioms zur Beschreibung theoretischer Praxis werden Robustheit und Resilienz als technische Kriterien für die Verlässlichkeit von Aussagen angeboten. ${ }^{23}$ Theorien müssen hiernach nicht wahr, wahrheitsähnlich oder empirisch adäquat sein, sie müssen nicht genau mit der Wirklichkeit übereinstimmen, keine präzisen Voraussagen machen oder reproduzierbare Bestätigungen erbringen. Es wird von ihnen nur verlangt, dass sie sich mit einer angemessenen Fehlertoleranz im Gebrauch bewähren. Dabei geht es entsprechend der wissenschaftsphilosophischen Leitfrage natürlich immer noch um Theorien oder Aussagen und nicht etwa um nicht-propositionale Vermögen oder Fertigkeiten - und dass sich Theorien im Gebrauch bewähren, soll Beleg oder Evidenz sein für ihre prädiktive Leistungsfähigkeit als Darstellungen der Wirklichkeit. ${ }^{24}$

Diese drei meines Erachtens symptomatischen Ansätze explizieren also jeweils noch die Vorstellung, dass die Wissenschaft auf die Darstellung von Phänomenen und Prozessen zielt. Insofern bedienen sich die jeweiligen Autoren eines technischen Vokabulars nur als Hilfsmittel für die Charakterisierung einer vorrangig theoretischen oder geistigen Leistung. Und doch erscheinen die so beschriebenen Wissenschaftler nicht mehr als Aufklärer, die große historische Entwicklungsprozesse des Reduktionismus, der Vereinheitlichung, der Rationalisierung befördern. Stattdessen liefern sie Stückwerk für die Bearbeitung spezifischer Probleme, die lokale Lösungen erfordern. In diesen Beschreibungen wissenschaftlicher Praxis wird schon so sehr auf die technischen Anforderungen der Herstellung von Übereinstimmung geachtet, dass das vorausgesetzte Wissens- und Wissenschaftsideal stillschweigend in der Bedeutungslosigkeit versinkt. Mit dem Verschwinden der großen Fragen von Rationalität und Realismus, geistigem Fortschritt oder wahrer Welterkenntnis bleibt ein so intimes Wechselspiel von Darstellung und Eingriff, „representing“ und „intervening“, dass die Unterscheidung von Wissenschaft und Technowissenschaft für redundant gehalten werden könnte: Selbstvergessen meint mancher Wissenschaftsphilosoph, aber auch mancher Historiker, dass wir um die Konfrontation unterschiedlicher, womöglich um kulturelle Dominanz ringender oder epochemachender Wissenschaftsbegriffe herumkämen, wenn wir mit einem diffusen Begriff von wissenschaftlicher Praxis meinten, schon alles erreicht zu

\footnotetext{
23 Vergleiche beispielsweise Sherrilyn Roush, Tracking Truth: Knowledge, Evidence, and Science, Oxford: Oxford University Press 2005.

24 Diese Liste beansprucht keine Vollständigkeit. Eine implizit technowissenschaftliche Einstellung der Wissenschaftsphilosophie charakterisiert beispielsweise auch den Bayesianismus und sein Instrumentarium zur Darstellung von Überzeugungsgraden und deren Veränderung.
} 
haben. ${ }^{25}$ Gegen diese Selbstvergessenheit tritt die Differenzierung von Wissenschaft und Technowissenschaft an. Da ist einerseits der latent weiterhin wirksame und darum explizit anzuerkennende Mythos einer der Aufklärung verpflichteten, hypothesenprüfenden Wissenschaft, die wahre oder empirisch adäquate Aussagen über die Wirklichkeit produziert. Da ist andererseits der gleichsam schon lange, nur nicht in der Wissenschaftsphilosophie wirksame Mythos einer der Innovation verpflichteten Technowissenschaft, die sich der Aneignung und dem Nachweis von Fertigkeiten der Phänomenkontrolle widmet. Tatsächlich dient die Artikulation des technowissenschaftlichen Forschungsideals auch der Selbstverständigung über den klassischen Wissenschaftsbegriff und der mit ihm verbundenen Werte. ${ }^{26}$

\section{TENDENZEN EINER TECHNOWISSENSCHAFTSPHILOSOPHIE}

Womit sich die heutige Wissenschaftsphilosophie in einem technischen Idiom beschäftigt, ist immer noch vornehmlich das Verhältnis von Geist und Welt, das die Wissenschaft in einen ideengeschichtlichen Zusammenhang stellt - auch wenn dieser Zusammenhang nicht mehr

25 Keinen diffusen Praxisbegriff, sondern zweierlei Kontinuität bringt Martin Carrier zur Geltung. Da ist einerseits die Kontinuität der Nutzenorientierung, die der gesamten modernen Wissenschaftsentwicklung zugrunde liegt. Da ist andererseits methodische Kontinuität in Bezug auf Kausalanalyse, Modellierung und Validierung. Phänomenkontrolle und Weltbeschreibung stünden somit immer in einer methodischen Wechselbeziehung. Die Erscheinung im 18. bis 20. Jahrhundert einer scheinbar rein theoretischen Wissenschaft dürfe von dieser Kontinuität nicht ablenken - siehe Martin Carrier, ,Knowledge is Power', or How to Capture the Relationship between Science and Technoscience, in: Alfred Nordmann, Hans Radder, Gregor Schiemann (Hrsgg.), Science Transformed? Debating Claims of an Epochal Break, Pittsburgh: Pittsburgh University Press 2011, S. 43-53. Damit unterschätzt Carrier meines Erachtens jedoch dreierlei: 1) die Wirkmacht einer auch noch so ephemeren Vorstellung von reiner Wissenschaft, insbesondere auf eine Wissenschaftsphilosophie, die ein ganz bestimmtes Selbstverständnis von Wissenschaft artikuliert und propagiert hat; 2) den Unterschied von Phänomenbeherrschung und Weltbeschreibung auch bezüglich Kausalanalyse, Modellierung und Validierung - diese Begriffe (wie die des Wissens, der Theorie, der Erklärung u.a.) bedeuten in den beiden Zusammenhängen etwas anderes; 3) die Entstehung neuer methodischer Gepflogenheiten auch jenseits der Kontinuität, etwa iterativer Verfahren zur Komplexitätssteigerung in Modellen oder Schussfolgerungen von der Ähnlichkeit zweier Visualisierungen auf eine ursächliche Dynamik. - Umgekehrt gebe ich gerne zu, dass ich Diskontinuität überschätze und vor allem nicht genügend würdige, dass die idealtypisch geschiedenen Forschungsmodi im Austausch miteinander stehen, sich korrigieren und ergänzen können.

26 So fasst Bernadette Bensaude-Vincent die Aufgabe einer Philosophie der Technowissenschaften auf, siehe Bernadette Bensaude-Vincent, Les vertiges de la technoscience. Façonner le monde atome par atome, Paris: La Découverte 2009. 
angesprochen wird, wo es um den lokalen Bezug von Theorie zu ihren Modellen geht, um die Mechanismen, die diese Modelle spezifizieren, und um die durch den Nachweis des HiggsBosons erwiesene Robustheit des Bezugs von Theorie und spezifizierter Wirklichkeit. Gekennzeichnet ist hiernach also die Geschichte der Wissenschaftsphilosophie der letzten 30 Jahre durch die Würdigung technischer Anforderungen bei der Darstellung von Wirklichkeit und durch den gleichzeitigen Rückzug von Fragestellungen, die noch mit Kuhn und Lakatos, Nagel und Putnam, Harman und Kitcher verbunden waren. Rationale Rekonstruktion und das Problem der Theorienwahl, Physikalismus und Reduktionismus, Vereinheitlichung und Schlüsse auf die beste Erklärung führen in gegenwärtigen Diskussionen allenfalls ein geisterhaftes Schattendasein in den Fachzeitschriften.

Diese gleichzeitigen Tendenzen bedeuten eine vielleicht skeptische, vielleicht durch die Wissenschaftsgeschichte ernüchterte Abkehr von der nur mehr ideologisch empfundenen Überhöhung des Fortschrittsglaubens, der unendlichen Wahrheitssuche, der kritischen wissenschaftlichen Weltanschauung, des Projekts Aufklärung. Und von hier ist es vielleicht nur noch ein kleiner Schritt zum radikalen Perspektivenwechsel, der den Forschungsprozess nicht als theoretische Weltdarstellung, sondern als technische Phänomenbeherrschung versteht und also das Vorurteil abstreift, demzufolge technische Phänomenbeherrschung immer subsidiär zur theoretischen Weltdarstellung sei. Vielmehr kann nun davon ausgegangen werden, dass das vorhandene Repertoire wissenschaftlicher Theorien in den großen Werkzeugkasten gehört, aus dem heraus neue Fertigkeiten der Phänomenbeherrschung erarbeitet werden. Inwieweit zeichnet sich nun bereits eine Technowissenschaftsphilosophie ab, die in diesem Sinne Wissenschaft als Technik versteht? Wiederum seien nur einige Entwicklungen benannt, die als Anhaltspunkte dienen können.

Da ist zunächst und vor allem die Philosophie der Computersimulation, wie sie von Paul Humphreys oder Johannes Lenhard verfolgt wird. ${ }^{27}$ Hier geht es um eine Forschungstechnologie, die überlieferte wissenschaftstheoretische Begriffe grundsätzlich in Frage stellt bzw. neu konfiguriert: Was ist ein Experiment, was ist ein Modell, was ist eine Erklärung, was heißt Verstehen, was ist das Verhältnis von Herstellen und Erkennen? Antworten auf diese Fragen lassen sich auch auf die Forschung mit Modellorganismen und den

\footnotetext{
27 Paul Humphreys, Extending Ourselves: Computational Science, Empiricism, and Scientific Method, Oxford: Oxford University Press 2004; Johannes Lenhard, Mit allem rechnen (wie Anm. 20) oder Johannes Lenhard, Günter Küppers, Terry Shinn (Hrsgg.), Simulation: Pragmatic Constructions of Reality, (Sociology of the Sciences Yearbook) Dordrecht: Springer 2007.
} 
von Astrid Schwarz und Wolfgang Krohn untersuchten „Realsimulationen“ übertragen. ${ }^{28}$ Die Tendenz, Tiermodelle oder Computermodelle für Darstellungen einer unabhängig gegebenen Wirklichkeit aufzufassen, lässt sich hier nicht durchhalten, weswegen diese Modelle nun als technische Systeme erscheinen, die durch ihren komplexitätssteigernden Konstruktionsprozess Wirklichkeit in sich aufnehmen, um die Wirklichkeit schließlich so weit substituieren zu können, dass Experimente an den Modellen für Experimente in der Wirklichkeit gehalten werden. ${ }^{29}$

Weiterhin wären hier in etwas eklektischer Reihung und bloß beispielhaft Arbeiten von Hasok Chang, Kenneth Waters, Maureen O’Malley, Robert Batterman oder Anna-Sophie Heinemann zu nennen. ${ }^{30}$ Chang betrachtet Metrologie und insbesondere Temperaturmessung und stößt dabei auf Verfahren der internen Validierung etwa durch Iteration. Iterative Verfahren finden sich im ,software engineering', in der Metrologie, der synthetischen Biologie und der Klimamodellierung. Hier wird gezeigt, wie sich ein technisches System, vielleicht im Zusammenspiel mit anderen technischen Systemen, durch iterative Anpassungen und Abstimmungen von Systemleistungen gewissermaßen selbst validiert, statt durch Konfrontation mit einer externen, logisch unabhängig konzipierten Wirklichkeit validiert zu werden. Nach klassisch-wissenschaftstheoretischer Vorstellung sind solche Verfahren unzulässig, weil zirkulär. Dabei wäre nun Aufgabe einer Technowissenschaftsphilosophie, die Rechtfertigung solcher Verfahren zu ergründen, gerade auch wenn man hierbei auf das Funktionieren der Technik verwiesen ist: Wie können wir verstehen, dass beispielsweise das bloße Vorzeigen und die Beschreibung eines Dings substanziell schon etwas beweist?31

\footnotetext{
${ }^{28}$ Astrid Schwarz, Wolfgang Krohn, Experimenting with the Concept of Experiment: Probing the Epochal Break, in: Nordmann, Radder, Schiemann, Science Transformed? (wie Anm. 25), S. 119-134; Evelyn Fox Keller, Models of and Models for: Theory and Practice in Contemporary Biology, Philosophy of Science 67 (2000), 72-86.

${ }^{29}$ Nordmann, Collapse of Distance (wie Anm. 9).

${ }^{30}$ Hasok Chang, Inventing Temperature: Measurement and Scientific Progress, Oxford: Oxford University Press 2007; C. Kenneth Waters, How Practical Know-How Contextualizes Theoretical Knowledge: Exporting Causal Knowledge from Laboratory to Nature, Philosophy of Science 75 (2008), 707-719; Maureen O'Malley, Exploration, Iterativity and Kludging in Synthetic Biology, Comptes Rendus Chimie 14 (2011), 406-412; Anna-Sophie Heinemann, ,... a visible and tangible proof that a new system of logical deduction has been attained': W. S. Jevons' ,Logisches Piano‘ als Methodenmo- dell, Vortrag beim XLVIII. Symposium der Gesellschaft für Wissenschaftsgeschichte, Wien, Mai 2011.

31 Hierher würde beispielsweise eine bisher nicht erbrachte wissenschaftsphilosophische Analyse des Machbarkeitsbeweises oder „proof of concept" gehören.
} 
Mit dem Verweis auf das bloße Ding kommt eine weitere Tendenz zur Sprache, die besonders allgemein und diffus auf eine Technowissenschaftsphilosophie abzielt. Nachdem Wissenschaftsphilosophen wie Cassirer oder Schlick die moderne Naturwissenschaft mit dem Verschwinden des Dinges zugunsten der Funktion oder der Tatsache gleichgesetzt hatten, fand in den letzten Jahren eine Renaissance des Dings statt, sowohl in wissenschaftshistorischen und -philosophischen Analysen als auch in den Technowissenschaften selbst. ${ }^{32}$ Nicht um Sinnesdaten, Relationen, Tatsachen geht es hier, sondern um die Dinge, ihre Eigenschaften, die Überraschungen, die sie bergen, ihre Ähnlichkeit. Mit welchem Recht lässt sich beispielsweise aus der Ähnlichkeit eines experimentell beobachteten und simulationsmodellierten Prozesses auf seine Erklärung schließen? Egal wie die Frage beantwortet wird, entspricht ihr eine Forschungspraxis, die rekonstruiert sein will.

\section{FreiE VerhäLtNisSe: PhILOSOPHIE UND GESCHICHTE DER TECHNOWISSENSCHAFTEN}

Nach dieser oberflächlichen und durchaus selektiven Bestandsaufnahme der heutigen Wissenschaftsphilosophie kündigt sich ein neues, technikphilosophisches Verständnis der Forschungspraxis und ihrer Kulturbedeutung bereits an, auch wenn es nur selten deutlich ausgesprochen wird. Wie eingangs versprochen gilt es abschließend noch zu bedenken, was dieses veränderte Verständnis für das Verhältnis von Philosophie und Geschichte der Wissenschaften bedeuten könnte. ${ }^{33}$

So lange Wissenschaft als Bemühung um theoretische Weltdarstellung vorgestellt wird, ist die Rolle der Wissenschaftsgeschichte in enger Abhängigkeit zur Philosophie definiert. Sie setzt sich institutionell und intellektuell von der allgemeinen Geschichte und anderen historischen Teildisziplinen ab - sie ist keine Technikgeschichte, Arbeits- und Sozialgeschichte oder Umweltgeschichte, sondern präsentierte sich zunächst als eine Spielart der Ideengeschichte, die zwischen die Räder des Realismus, Relativismus und Konstruktivismus geriet und einen Mittelweg zwischen internalistischer und externalistischer Geschichtsschreibung, auch zwischen „whig-history“ und Bedeutungsholismus suchte. Eng mit philosophischen Debatten

\footnotetext{
32 Bensaude-Vincent, Loeve, Nordmann, Schwarz, Matters of Interest (wie Anm. 19).

33 Die folgenden Bemerkungen sind stark von Ann Johnson, Historikerin an der University of South Carolina, beeinflusst.
} 
verflochten, beschäftigte sich Wissenschaftsgeschichte mit Kontroversen und Umbrüchen, wurde eine Geschichte der Instrumente und Labore - je nachdem wie die Einflussgrößen auf Theoriebildung und das Geschehen im Labor diskutiert und gedacht wurden.

Ein freieres Verhältnis von Philosophie und Geschichte der Wissenschaften ergibt sich einerseits, wenn Wissenschaftsgeschichte eigene, beispielsweise kulturwissenschaftlich orientierte Wege verfolgt, wenn sie sich also gar nicht auf Wissenschaftsphilosophie bezieht, allenfalls gerne von der Philosophie entdecken lässt. ${ }^{34}$ Ein freieres Verhältnis kann sich aber auch dort ergeben, wo der Bezug erhalten bleibt, Wissenschaft aber als technisches Handeln in einer Welt vorgestellt wird, die schon das Produkt wissenschaftlich-technischer Entwicklungen ist und die auf diese Welt verändernd zurückwirkt. Dies spricht insbesondere für Umweltgeschichte als Wissenschafts- und Technikgeschichte, somit eine Geschichtsschreibung, die thematisieren kann, wann Forschung aufhört und beginnt, wie sie Bedeutsamkeit erlangt im Austausch mit zahlreichen Akteuren und vielfältigen Interessen, die zur immer auch wissenschaftlich-technischen Veränderung der Welt beitragen. Diese Art von Wissenschaftsgeschichte wird darum nicht etwa die Bedeutung von Wissenschaft und Technowissenschaft unterschätzen, sondern auf Wissensproduktion bezogen bleiben, wobei dieses Wissen aber nicht auf theoretische Weltbeschreibung beschränkt wird. Hier handelt es sich nun um ein Wissen, das die Welt nicht erst in der technischen Anwendung verändert, sondern schon durch Problemdefinition, Gegenstandsbezug, die Aneignung neuer Fertigkeiten. ${ }^{35}$

Der Übergang von einem engeren, nämlich wohldefinierten zu einem freieren, nämlich unbefangenen Verhältnis von Wissenschaftsphilosophie und Wissenschaftsgeschichte hängt eng mit dem Begriff bzw. dem Selbstverständnis der Forschung zusammen, das es philosophisch und historisch zu artikulieren gilt. ${ }^{36}$ Insofern es der Wissenschaft darum geht,

\footnotetext{
34 Eine weitere produktive Spielart ergibt sich, wenn sich Wissenschaftshistoriographie bewusst auf andere Traditionen der Wissenschaftsphilosophie bezieht, beispielsweise auf Bachelard, Canguilhem, Foucault, Latour und das Projekt einer historischen Epistemologie.

35 Hier spätestens müsste Bruno Latour genannt werden, der wie Francis Bacon oder Martin Heidegger im Zusammentreffen von Wissenschafts- und Technikphilosophie vom Außenseiter zum Mitdenker avanciert. Dass Forschung die Welt verändert, schon indem die Experimentatoren und ihre Forschungsgegenstände gleichzeitig neue Kompetenzen erwerben, verdeutlicht er vorbildlich in The Force and the Reason of Experiment, in: Homer LeGrand (Hrsg.), Experimental Inquiries, Dordrecht: Kluwer 1990, S. 49-80.

36 Für die oben bereits diskutierte „selbstvergessene“ Wissenschaftsphilosophie wie für eine ganz von dieser Wissenschaftsphilosophie losgelöste Wissenschaftshistoriographie gilt gleichermaßen, dass sie oftmals nur implizit mit der
} 
eine geistesunabhängig gegebene oder gedachte Wirklichkeit darzustellen, richtet sich ihre ganze Anstrengung gegen den Anschein historischer Kontingenz. Es muss der Erweis erbracht werden für das, was Paul Feyerabend die „separability assumption“ genannt hat, also für die Annahme, dass die Wissenschaft Aussagen über die Wirklichkeit machen kann, die unabhängig von ihren Entstehungsbedingungen allgemein gelten. ${ }^{37}$ Damit steht von vornherein fest, dass Wissenschaftsgeschichtsschreibung immer nur das eine oder das andere leisten kann: Entweder sie beschränkt sich brav darauf nachzuzeichnen, wie die Wissenschaft das macht und wie sie ihre Geltungsansprüche entwickelt, weitgehend losgelöst von den Veränderungen der Arbeitsverhältnisse oder der Rohstoffnutzung oder der Bildungssysteme, der politischen Revolutionen und Kriege (und wenn derlei überhaupt ins Spiel kommt, dann vor allem als Störfaktor, als womöglich hartnäckige Präsenz einer gesellschaftlichen Wirklichkeit, die es auch noch zu berücksichtigen gilt) - oder die Wissenschaftsgeschichtsschreibung stellt sich gegen den Anspruch der Wissenschaft selbst und weist ihr immerfort nach, dass sie die „separability assumption" nicht eingelöst hat und dass ihre Theoriebildungen selbstverständlich noch die Spuren ihres Entstehungszusammenhangs tragen.

So zwingend dieses Entweder-oder ist, so bezeichnend ist doch auch, dass diese Alternative nicht mehr zeitgemäß erscheint, sondern nur mehr die Erinnerung aufruft an heftige Debatten, in denen die Übereinstimmung von Theorie und Wirklichkeit realistisch und konstruktivistisch, internalistisch und externalistisch ganz unterschiedlich gedeutet wurde und in denen die Möglichkeit dieser Übereinstimmung noch im Zentrum des Interesses stand. Wenn dieses Entweder-oder für die Praxis der Wissenschaftsgeschichtsschreibung nicht mehr zu gelten scheint, so ist dies ein weiterer Beleg dafür, dass die Idee einer auf theoretische Weltbeschreibung zielenden Wissenschaft weitgehend abgelöst wurde von einer weniger deutlich artikulierten, aber für das jetzt vorherrschende Verständnis offenbar tragfähigeren Vorstellung von Wissenschaft als Technik der Phänomenbeherrschung und Weltveränderung. Wo nun dieses etwas diffuse, technowissenschaftliche Verständnis von Forschungspraxis vorherrscht, kehrt sich das Abhängigkeitsverhältnis tendenziell um. Ganz selbstverständlich

\footnotetext{
Artikulation eines Wissenschaftsbegriffs befasst sind, schon weil sie die Idee eines „Wissenschaftsbegriffs" misstrauisch stimmt. Nun ist sowohl der Begriff von Wissenschaft als auch der Begriff von Technowissenschaft nicht etwa mit der Behauptung verknüpft, es gebe „die eine Wissenschaft“ und „die eine Technowissenschaft". Vielmehr fungieren diese Begriffe wie „Aufklärung“ oder „Moderne“, „Wissensgesellschaft" oder „Nachhaltigkeit“ - es sind Begriffe, die die Forschungspraxis informieren, von ihr aufgefasst und transformiert werden und gerade darum artikuliert werden müssen.

37 Paul Feyerabend, Realism and the Historicity of Knowledge, in: derselbe, Conquest of Abundance, hrsg. von Bert Terpstra, Chicago: University of Chicago Press 2001, S. 131-146.
} 
fügt sich nun Forschung in die Zusammenhänge einer von Wissenschaft und Technik längst durchwirkten Welt und trägt zu ihrer weiteren Veränderung bei. Egal wo wir hinschauen, kann die Wissenschaftsgeschichte einen Beitrag leisten - industrielle Produktionsverfahren, soziale Netzwerke, Waldsterben, Wertpapierhandel, humanoide Roboter, Flüssigkristalle verdanken sich dem Zusammenspiel zahlreicher Faktoren, einschließlich den Analysemethoden, Prüfverfahren, Theorien und Modellen einer akademisch grundlagenorientierten Forschung. Gleichzeitig erweitert sich die Frage nach dem, was Wissenschaft ist, zur Frage nach dem Funktionieren einer Wissensgesellschaft, die aus Realexperimenten lernt, die Risiken abschätzt, die den Kohlendioxidausstoß reduziert, die eine Energiewende vollzieht. Analog den Regierungen und Verwaltungsbehörden, analog der Gesetzgebung und Rechtsprechung konzentrieren sich in Universitäten und Forschungslaboren gesellschaftliche Problemstellungen und werden dort auf eine diesen Institutionen spezifische Weise verhandelt - dies ist historisch aufschlussreich und begründet die zentrale Bedeutung von Wissenschaftsund Technikgeschichte für ein intimes Verständnis der Wissensgesellschaften.

Was diese Perspektivierung wissenschaftlicher und technowissenschaftlicher Forschung aus dem Blick verliert, ist die Vorstellung einer privilegierten Entwicklungsdynamik, die sich aufklärerisch an dem Ideal der kritischen Wahrheitssuche orientiert und dieses Ideal nicht nur gegen Aberglauben und Vorurteil behauptet, sondern auch gegen gesellschaftliche Ansprüche auf Nutzen, Innovation oder technische Phänomenbeherrschung zur Geltung bringt. Ein solcher Eigensinn der Wissenschaft wird nun als ideologische Überhöhung und bloße Selbststilisierung historisiert und relativiert. Je weniger glaubwürdig wir den aufklärerischen Anspruch der Wissenschaft finden und je leichter es uns fällt, überall immer nur technische Leistungen zu sehen, desto deutlicher tritt die eingangs erwähnte Ambivalenz hervor: Zu prägend war die Allianz von Wissenschaft und Aufklärung, die an den Beispielen von Galileo, Darwin, Freud und Einstein immer noch aufgerufen wird, als dass sie reduziert werden könnte auf das bloße Selbstmissverständnis einer bei genauerem Hinsehen immer nur anwendungsbezogenen Wissenschaft. Schon um die Kulturbedeutung des klassischen, hier und heute in Frage gestellten Wissenschaftsbegriffs anzuerkennen, ziehe ich es einem solchen Reduktionismus gegenüber vor, mit lachendem und weinendem Auge einen Epochenbruch zu behaupten, demnach die Desillusionierung von Aufklärung und Wissenschaft zwar den Blick auf Wissenschaft als Technik freilegt, damit aber auch unverzichtbare, ihrerseits wirkmächtige 
Erkenntnisideale preisgibt. ${ }^{38}$ Aber die Problematik eines Epochenbruchs und was damit gemeint sein dürfte ist, in jeder Hinsicht, eine andere Geschichte.

\section{KONTAKT}

\section{Alfred Nordmann}

Technische Universität Darmstadt

nordmann@phil.tu-darmstadt.de

38 Paul Forman, The Primacy of Science in Modernity, of Technology in Postmodernity, and of Ideology in the History of Technology, History and Technology 23 (2007), 1-152; Alfred Nordmann, The Age of Technoscience, in: Nordmann, Radder, Schiemann, Science Transformed? (wie Anm. 25), Pittsburgh: Pittsburgh University Press 2011, S. 19-30. 